RAIRO Operations Research

RAIRO Oper. Res. 41 (2007) 49-59

DOI: $10.1051 /$ ro:2007006

\title{
A NUMERICAL FEASIBLE INTERIOR POINT METHOD FOR LINEAR SEMIDEFINITE PROGRAMS
}

\author{
Djamel Benterki ${ }^{1}$, Jean-Pierre Crouzeix ${ }^{2}$ \\ AND BACHIR MERIKHI ${ }^{1}$
}

\begin{abstract}
This paper presents a feasible primal algorithm for linear semidefinite programming. The algorithm starts with a strictly feasible solution, but in case where no such a solution is known, an application of the algorithm to an associate problem allows to obtain one. Finally, we present some numerical experiments which show that the algorithm works properly.
\end{abstract}

Keywords. Linear programming, semidefinite programming, interior point methods.

Mathematics Subject Classification. 90C51, 90C22, 90C05.

\section{INTRODUCTION}

The aim of this paper is to present a feasible interior point method for the linear semidefinite program:

$$
z^{*}=\min _{X}\left[\langle C, X\rangle: X \in K,\left\langle A_{i}, X\right\rangle=b_{i} \text { for } i=1, \ldots, m\right] .
$$

Here $b \in \mathbb{R}^{m}, K$ denotes the cone of positive semidefinite matrices in the linear space of $n \times n$ symmetric matrices $E$. The matrices $C$ and $A_{i}, i=1, \ldots, m$, are given and belong to $E$. The inner product on $E$ of two matrices $A$ and $B$ is the trace of their product, i.e., $\langle A, B\rangle=\operatorname{tr}(A B)=\sum_{i, j} a_{i j} b_{i j}$. It is known that the interior of $K$, denoted by $\operatorname{int}(\mathrm{K})$, is the set of positive definite matrices of $E$.

Received March 3, 2005. Accepted September 29, 2006.

${ }^{1}$ Département de Mathématiques, Faculté des sciences, Université Ferhat Abbas, Sétif, 19000,

Algérie ; dj_benterki@yahoo.fr; b_merikhi@yahoo.fr

2 LIMOS, Université Blaise Pascal, 63177 Aubière Cedex, France;

jp.crouzeix@math.univ-bpclermont.fr

(C) EDP Sciences, ROADEF, SMAI 2007 
In references $[5,11]$, the reader will find a description of a few applications of linear semidefinite programming, in particular, max-cut problems in a graph, graph-bisection problems, the search of a largest clique in a graph, min-max eigenvalue problems.

Linear semidefinite programming presents a great similarity with linear programming: the objective function is linear, the constraint functions are affine, the difference consists in the positive cone. Indeed, testing the positive semidefiniteness of an $n \times n$ matrix $X$ requires to check $\langle X h, h\rangle \geq 0$ for all $h$ with norm 1 , an infinity of linear constraints, while testing that a vector belongs to the nonnegative orthant of an Euclidean space involves, by definition, a finite number of linear constraints. The duality schemes present also similarities, one main difference is that for the strong duality result in linear semidefinite programming the primal and the dual problems have to be strictly feasible instead of simply feasible in classical linear programming. Besides, on an algorithmical point of view, interior point methods used in linear programming can be easily extended to linear semidefinite programming. Most of the algorithms $[2,3,5,6]$ are extensions of path following or related methods in linear programming to $S D P$ programming using a Newton descent direction.

Our algorithm is close to the projective algorithm of Alizadeh [1] (for more informations on projective methods, see $[4,7,8]$ ). As in the Alizadeh algorithm, the descent direction is obtained by the projection on a linear subspace, but in our presentation the computations appear to be simpler, in particular our algorithm does not make use of a potential function. This simplicity has a price since potential functions are commonly used in interior point methods to prove the theoretical convergence of algorithms. Still, the numerical experiments show the good behaviour of our algorithm.

As many interior point methods, the algorithm needs the knowledge of an initial strictly feasible solution. In case no such a solution is available, a first application of the algorithm allows to get one.

Now, we make precise the notation used in the paper. We have already defined the sets $E, K, \operatorname{int}(\mathrm{K})$ and the scalar product $\langle$,$\rangle in E$. The identity matrix of $E$ is denoted by $I$. Given $A \in E$ its norm is

$$
\|A\|=\sqrt{\langle A, A\rangle}=\left(\sum_{i=1}^{n}\left|\lambda_{i}\right|^{2}\right)^{\frac{1}{2}}
$$

where $\lambda_{i}, i=1,2, \ldots, n$ are the eigenvalues of $A$.

\section{The DUAlity IN SEMIDEFinite PROGRAMMing}

Let us consider the problems:

$$
z^{*}=\min _{X}\left[\langle C, X\rangle \quad: \quad X \in K,\left\langle A_{i}, X\right\rangle=b_{i} \text { for } i=1, \ldots, m\right]
$$


and

$$
m_{d}=\max _{w}\left[b^{t} w \quad: \quad\left(C-\sum_{i=1}^{m} w_{i} A_{i}\right) \in K\right] .
$$

A matrix $X$ is said to be a strictly feasible solution of $(S D P)$ if it belongs to int $(\mathrm{K})$ and $\left\langle A_{i}, X\right\rangle=b_{i}$, for $i=1, \ldots, m$, a vector $w \in \mathbb{R}^{m}$ is said to be a strictly feasible solution of $(D S D P)$ if the matrix $\left(C-\sum_{i=1}^{m} w_{i} A_{i}\right) \in \operatorname{int}(\mathrm{K})$. The weak duality result says that we have always $m_{d} \leq z^{*}$. The strong duality result says that if both $(S D P)$ and $(D S D P)$ have strictly feasible solutions, then $m_{d}=z^{*}$ and both problems have optimal solutions. Furthermore, for such optimal solutions $X$ and $w$, the following complementarity slackness condition holds

$$
\left\langle X, C-\sum_{i=1}^{m} w_{i} A_{i}\right\rangle=0 .
$$

Also, the sets of optimal solution of $(S D P)$ and $(D S D P)$ are closed convex and bounded.

More information on semidefinite programming and its duality can be found in references [9-11].

\section{Description of the Algorithm}

Throughout the paper, we made the following assumptions.

(1) $C \neq 0$ and the constraints $\left\langle A_{i}, X\right\rangle=b_{i}$ for $i=1, \ldots, m$ are not redundant.

(2) Both problems $(S D P)$ and $(D S D P)$ have strictly feasible solutions.

In this section, we describe the passage from an iterate $X_{k}$ to the next one $X_{k+1}$. The problem of finding an initial feasible solution $X_{0}$ will be considered in another section.

At the beginning of step $\mathrm{k}$, the current $X_{k}$ is a strictly feasible solution of $(S D P)$. The Cholesky factorization of the positive definite matrix $X_{k}$ gives a lower triangular matrix $L_{k}$ with positive diagonal entries such that $L_{k} L_{k}^{t}=X_{k}$. Then, we define the projective transformation

$$
T_{k}(X)=(Y, \alpha)
$$

where

$$
Y=\alpha L_{k}^{-1} X L_{k}^{-t} \quad, \quad \alpha=\frac{(n+1)}{1+\left\langle X_{k}^{-1}, X\right\rangle} .
$$

The transformation $T_{k}$ is one to one from $K$ to $\widetilde{K}$ where

$$
\widetilde{K}=\{(Y, \alpha) \in K \times(0,+\infty):\langle I, Y\rangle+\alpha=n+1\}
$$


The inverse transformation $T_{k}^{-1}$ is such that

$$
T_{k}^{-1}(Y, \alpha)=\frac{1}{\alpha} L_{k} Y L_{k}^{t} .
$$

For simplicity, we introduce the matrices:

(1) $C_{k}=L_{k}^{t} C L_{k}$.

(2) $A_{i}^{(k)}=L_{k}^{t} A_{i} L_{k}$ for $i=1, \ldots, m$.

Then, for $(Y, \alpha)=T_{k}(X)$, we have:

$$
\left\langle C_{k}, Y\right\rangle-z^{*} \alpha=\alpha\left[\langle C, X\rangle-z^{*}\right] .
$$

Furthermore, the constraints $\left\langle A_{i}, X\right\rangle=b_{i}$ for $i=1, \ldots, m$ and $X \in K$ are equivalent to the conditions:

$$
\left\{\begin{array}{l}
\left\langle A_{i}^{(k)}, Y\right\rangle-\alpha b_{i}=0, i=1, \ldots, m \\
\langle I, Y\rangle+\alpha=n+1, \\
Y \in K, \alpha \geq 0 .
\end{array}\right\}
$$

It results that solving $(S D P)$ is equivalent to solving the problem:

$$
0=\min _{(Y, \alpha)}\left[\begin{array}{ll}
\left.\left\langle C_{k}, Y\right\rangle-z^{*} \alpha: \begin{array}{l}
(k) \\
: Y
\end{array}\right) & \langle I, Y\rangle+\alpha b_{i}=0, i=1, \ldots, m \\
Y \in K, \alpha \geq 0 .
\end{array}\right]
$$

Notice that $(I, 1)$ is a strictly feasible solution of problem $\left(E_{k}^{*}\right)$. Because the value $z^{*}$ is unknown, we consider its approximation

$$
z_{k}=\left\langle C, X_{k}\right\rangle=\left\langle C_{k}, I\right\rangle .
$$

Then $z_{k}>z^{*}$. Next, we consider the semidefinite program

$$
m_{k}=\min _{(Y, \alpha)}\left[\begin{array}{ll} 
& \left\langle A_{i}^{(k)}, Y\right\rangle-\alpha b_{i}=0, i=1, \ldots, m \\
\left\langle C_{k}, Y\right\rangle-z_{k} \alpha: & \langle I, Y\rangle+\alpha=n+1 \\
Y \in K, \alpha \geq 0 .
\end{array}\right]
$$

In a similar way to Karmarkar's method for classical linear programs, we relax problem $\left(E_{k}\right)$ into the convex optimization problem

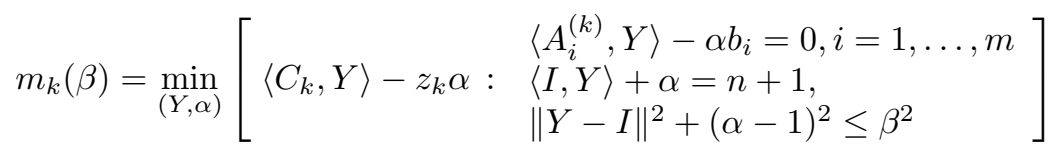

with $\beta>0$. Here again, $(I, 1)$ is a strictly feasible solution and therefore $m_{k}(\beta) \leq$ 0 . Moreover, if $\beta \in(0,1)$, the feasible set of $\left(E_{k}^{r}\right)$ is contained in the feasible set 
of $\left(E_{k}\right)$ and then $m_{k}(\beta) \geq m_{k}$. Let us turn our interest to the function $m_{k}$. It is clear that if $0<\beta<\beta^{\prime}$ we have

$$
0 \geq m_{k}(\beta) \geq m_{k}\left(\beta^{\prime}\right) .
$$

The next proposition shows that the function $m_{k}$ is actually strictly negative on $(0,+\infty)$.

\section{Proposition 3.1.}

$$
m_{k}(\beta)<0 \text { for all } \beta>0 .
$$

Proof.Assume, for contradiction, that $m_{k}(\beta)=0 .(I, 1)$ being a feasible solution of $\left(E_{k}^{r}\right)$, is optimal too. Apply the first order optimality condition: there exist $\lambda \in \mathbb{R}^{m}$ and $\mu$ such that

$$
C_{k}+\sum_{i=1}^{m} \lambda_{i} A_{i}^{(k)}+\mu I=0 \quad \text { and } \quad-z_{k}-\sum_{i=1}^{m} b_{i} \lambda_{i}+\mu=0 .
$$

Since

$$
C_{k}=L_{k}^{t} C L_{k} \quad \text { and } \quad A_{i}^{(k)}=L_{k}^{t} A_{i} L_{k}
$$

the first equation is equivalent to

$$
C=-\sum_{i=1}^{m} \lambda_{i} A_{i}-\mu X_{k}^{-1}
$$

It follows that:

$$
\begin{aligned}
& z_{k}=\left\langle C, X_{k}\right\rangle=-\sum_{i=1}^{m} \lambda_{i}\left\langle A_{i}, X_{k}\right\rangle-\mu n \\
& z_{k}=-\sum_{i=1}^{m} b_{i} \lambda_{i}-\mu n .
\end{aligned}
$$

Hence $\mu=0$ and therefore

$$
z_{k}=-\sum_{i=1}^{m} b_{i} \lambda_{i} \quad \text { and } \quad C+\sum_{i=1}^{m} \lambda_{i} A_{i}=0 .
$$

Thus, $-\lambda$ is a feasible solution of $(D S D P)$ and therefore

$$
z_{k}=\sum_{i=1}^{m} b_{i}\left(-\lambda_{i}\right) \leq m_{d}=z^{*}<z_{k}
$$

which is not possible.

Set $V=Y-I$ and $v=\alpha-1$, the problem $\left(E_{k}^{r}\right)$ is equivalent to getting the optimal solution of the convex optimization problem

$$
\min _{(V, v)}\left[\begin{array}{ll} 
& \left\langle A_{i}^{(k)}, V\right\rangle-b_{i} v=0, i=1, \ldots, m, \\
\left\langle C_{k}, V\right\rangle-z_{k} v: & \langle I, V\rangle+v=0, \\
& \|V\|^{2}+v^{2} \leq \beta^{2} .
\end{array}\right]
$$


In view of the necessary and sufficient condition for optimality, the problem consists in finding $(V, v, \lambda, \mu, t) \in E \times \mathbb{R} \times \mathbb{R}^{m} \times \mathbb{R} \times[0,+\infty[$ such that:

$$
\begin{gathered}
C_{k}+\sum_{i=1}^{m} \lambda_{i} A_{i}^{(k)}+\mu I+t V=0 \\
-z_{k}-\sum_{i=1}^{m} b_{i} \lambda_{i}+\mu+t v=0 \\
\left\langle A_{j}^{(k)}, V\right\rangle-b_{j} v=0 ; \quad j=1, \ldots, m \\
\langle I, V\rangle+v=0 \\
t\left(\|V\|^{2}+v^{2}-\beta^{2}\right)=0 \\
\|V\|^{2}+v^{2} \leq \beta^{2} .
\end{gathered}
$$

Note that one has necessarily $t>0$, (if not $(V, v)=(0,0)$ would be an optimal solution of $\left.\left(E_{k}^{t}\right)\right)$. Hence, from (1) and (2), we get

$$
V=-t^{-1} V_{k} \text { and } v=-t^{-1} v_{k}
$$

where

$$
V_{k}=C_{k}+\sum_{i=1}^{m} \lambda_{i} A_{i}^{(k)}+\mu I \text { and } v_{k}=-\sum_{i=1}^{m} b_{i} \lambda_{i}+\mu-z_{k} .
$$

By construction, $V_{k}$ and $V$ are symmetric. Replacing $V$ and $v$ in (3) and (4), we obtain

$$
\mu=0
$$

and $\lambda$ is a solution of the $m \times m$ linear system

$$
M \lambda=d
$$

where for $i, j=1, \ldots, m$

$$
\begin{aligned}
& M_{i j}=\left\langle A_{i}^{(k)}, A_{j}^{(k)}\right\rangle+b_{i} b_{j} \\
& d_{i}=-b_{i} z_{k}-\left\langle C_{k}, A_{i}^{(k)}\right\rangle .
\end{aligned}
$$

By construction, $M$ is symmetric positive semidefinite. It is also positive definite by assumption 1 . Hence the system (7) has one solution which can be obtained via the Cholesky method. Thus $V_{k}$ and $v_{k}$ are easily obtained.

Next, the optimal solution $(V, v)$ of $\left(E_{k}^{t}\right)$ is given by

$$
V=-\beta P_{k} \text { and } v=-\beta p_{k}
$$

where

$$
P_{k}=\frac{V_{k}}{\tau}, \quad p_{k}=\frac{v_{k}}{\tau} \quad \text { and } \quad \tau=\left(\left\|V_{k}\right\|^{2}+v_{k}^{2}\right)^{\frac{1}{2}}
$$


Let us return to problem $\left(E_{k}^{r}\right)$. We see that the optimum is reached for

$$
Y(\beta)=I-\beta P_{k} \quad \text { and } \quad \alpha(\beta)=1-\beta p_{k} .
$$

We choose $\beta$ in such a way that the matrix $Y(\beta)$ stays positive definite and the scalar $\alpha(\beta)$ stays positive. Then, the next iterate $X_{k+1}$ is obtained by the formula:

$$
X_{k+1}=T_{k}^{-1}(Y(\beta), \alpha(\beta)) .
$$

It is clear that $X_{k+1}$ is a strictly feasible solution for $(S D P)$. Besides, the matrices $V_{k}, P_{k}, Y(\beta)$ and $X_{k+1}$ are symmetric.

The next proposition gives an easily checked criteria in order that $X_{k+1}$ stays in the strictly feasible solution set of $(S D P)$.

Proposition 3.2. Define

$$
\tilde{\beta}_{k}=\left[\max \left(p_{k}, \bar{\lambda}+\sigma \sqrt{n-1}\right)\right]^{-1}
$$

where

$$
\bar{\lambda}=\frac{1}{n} \sum_{i=1}^{n}\left(P_{k}\right)_{i i} \quad \text { and } \sigma=\frac{1}{n} \sum_{i=1}^{n} \sum_{j=1}^{n}\left(P_{k}\right)_{i j}^{2}-\bar{\lambda}^{2} .
$$

Then $X_{k+1}=T_{k}^{-1}(Y(\beta), \alpha(\beta))$ is a strictly feasible solution of $(S D P)$ for any $\beta \in\left(0, \tilde{\beta}_{k}\right)$ if $\tilde{\beta}_{k}>0$ and for any $\beta>0$ otherwise.

Proof. We must prove that the matrix $Y(\beta)$ is positive definite and the scalar $\alpha(\beta)$ is positive.

Let us denote by $\lambda_{1}, \lambda_{2}, \ldots, \lambda_{n}$ the eigenvalues of the matrix $P_{k}$, then from [12], we have

$$
\begin{aligned}
& \bar{\lambda}-\sigma \sqrt{n-1} \leq \min _{i} \lambda_{i} \leq \bar{\lambda}-\frac{\sigma}{\sqrt{n-1}}, \\
& \bar{\lambda}+\frac{\sigma}{\sqrt{n-1}} \leq \max _{i} \lambda_{i} \leq \bar{\lambda}+\sigma \sqrt{n-1} .
\end{aligned}
$$

It follows that $Y(\beta)=I-\beta P_{k}$ is positive definite when

$$
1-\beta \max _{i} \lambda_{i}>0
$$

i.e., when

$$
\frac{1}{\beta}>\bar{\lambda}+\sigma \sqrt{n-1}
$$

On the other hand, $\alpha(\beta)=1-\beta p_{k}$ is strictly positive when

$$
\frac{1}{\beta}>p_{k} .
$$

Summarizing, $X_{k+1}$ is strictly feasible when $0<\beta<\tilde{\beta}_{k}$.

The next proposition shows that for $\beta \in\left(0, \tilde{\beta}_{k}\right)$, we obtain a reduction of the value of the objective function of $(S D P)$. 
Proposition 3.3. For any $\beta \in\left(0, \tilde{\beta}_{k}\right)$, it holds

$$
\begin{gathered}
X_{k+1}=X_{k}-\frac{\beta}{1-\beta p_{k}}\left(L_{k} P_{k} L_{k}^{t}-p_{k} X_{k}\right), \\
m_{k}(\beta)=-\beta\left[\left\langle C_{k}, P_{k}\right\rangle-z_{k} p_{k}\right],
\end{gathered}
$$

and

$$
\left\langle C, X_{k+1}\right\rangle-\left\langle C, X_{k}\right\rangle=\frac{1}{1-\beta p_{k}} m_{k}(\beta)<0 .
$$

Proof. Replacing $Y(\beta)$ and $\alpha(\beta)$ in the formula

$$
X_{k+1}=T_{k}^{-1}(Y(\beta), \alpha(\beta))=\frac{1}{\alpha(\beta)} L_{k} Y(\beta) L_{k}^{t},
$$

we obtain

$$
\begin{aligned}
X_{k+1} & =X_{k}-\frac{\beta}{1-\beta p_{k}} L_{k}\left(P_{k}-p_{k} I\right) L_{k}^{t}, \\
& =X_{k}-\frac{\beta}{1-\beta p_{k}}\left(L_{k} P_{k} L_{k}^{t}-p_{k} X_{k}\right) .
\end{aligned}
$$

On the other hand,

$$
\begin{aligned}
m_{k}(\beta) & =\left\langle C_{k}, Y(\beta)\right\rangle-z_{k} \alpha(\beta), \\
& =\left\langle C_{k}, I\right\rangle-z_{k}-\beta\left[\left\langle C_{k}, P_{k}\right\rangle-z_{k} p_{k}\right], \\
& =\left\langle C, X_{k}\right\rangle-z_{k}-\beta\left[\left\langle C_{k}, P_{k}\right\rangle-z_{k} p_{k}\right], \\
& =-\beta\left[\left\langle C_{k}, P_{k}\right\rangle-z_{k} p_{k}\right] .
\end{aligned}
$$

It follows that

$$
\left\langle C, X_{k+1}\right\rangle-\left\langle C, X_{k}\right\rangle=\frac{1}{1-\beta p_{k}} m_{k}(\beta) .
$$

We know by Proposition 3.1 that $m_{k}(\beta)<0$ for all $\beta>0$.

Now, we summarize the algorithm.

\section{Description of the algorithm}

\section{a) Initialization:}

(1) $k=0, X_{0}$ is a strictly feasible solution of the problem.

(2) We choose a parameter $\rho \in(0,1)$ and a small $\varepsilon>0$ (for the stopping rule).

b) Step k: At the beginning of the step, $X_{k}$ is a strictly feasible solution of $(S D P)$.

(1) Set $z_{k}=\left\langle C, X_{k}\right\rangle$.

(2) Determine $L_{k}$ such that $X_{k}=L_{k} L_{k}^{t}$. Next, compute
(a) $C_{k}=L_{k}^{t} C L_{k}$,
(b) $A_{i}^{(k)}=L_{k}^{t} A_{i} L_{k}, \quad i=1, \ldots, m$.

(3) Compute the matrix $M$ and the vector $d$ as:
(a) $M_{i j}=\left\langle A_{i}^{(k)}, A_{j}^{(k)}\right\rangle+b_{i} b_{j}, \quad i, j=1, \ldots, m$,
(b) $d_{i}=-b_{i} z_{k}-\left\langle C_{k}, A_{i}^{(k)}\right\rangle i=1, \ldots, m$. 
(4) Solve the linear system $M \lambda=d$.

(5) Compute
(a) $V_{k}=\left(C_{k}+\sum_{i=1}^{m} \lambda_{i} A_{i}^{(k)}\right)$,
(b) $v_{k}=\left(-\sum_{i=1}^{m} b_{i} \lambda_{i}-z_{k}\right)$,
(c) $\tau=\left(\left\|V_{k}\right\|^{2}+v_{k}^{2}\right)^{\frac{1}{2}}$,
(d) $\bar{\lambda}=\frac{1}{n} \sum_{i=1}^{n}\left(P_{k}\right)_{i i}$,
(e) $\sigma=\frac{1}{n} \sum_{i=1}^{n} \sum_{j=1}^{n}\left(P_{k}\right)_{i j}^{2}-\bar{\lambda}^{2}$,
(f) $\beta_{k}=\rho\left[\max \left(p_{k}, \bar{\lambda}+\sigma \sqrt{n-1}\right)\right]^{-1}$.

(6) Compute
(a) $X_{k+1}=X_{k}-\frac{\beta_{k}}{\tau-\beta_{k} v_{k}} L_{k}\left(V_{k}-v_{k} I\right) L_{k}^{t}$.

(7) Stopping rule

(a) If $\beta_{k}\left[\left\langle C_{k}, V_{k}\right\rangle-z_{k} v_{k}\right]<\tau \varepsilon$ : STOP,

(b) if not, do $k=k+1$ and go back to Step $\mathbf{k}$.

\section{Finding AN INITIAL FEASIBLE SOLUTION}

The strict feasibility problem of $(S D P)$ consists of finding a $n \times n$ matrix $X$ such that:

$$
X \in \operatorname{int}(\mathrm{K}), \quad\left\langle A_{i}, X\right\rangle=b_{i} \text { for } i=1, \ldots, m .
$$

In order to solve this problem, we introduce the linear semidefinite program:

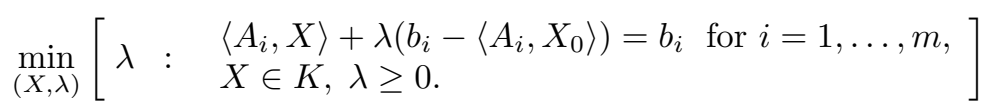

Then $X^{*}$ is a solution of problem $(F)$ if and only if $\left(X^{*}, 0\right)$ is an optimal solution of problem $(A P)$ and $X^{*} \in \operatorname{int}(\mathrm{K})$.

Note that $(A P)$ can be reformulated as:

$$
\min _{X^{\prime}}\left[\left\langle C^{\prime}, X^{\prime}\right\rangle: X^{\prime} \in K,\left\langle A_{i}^{\prime}, X^{\prime}\right\rangle=b_{i} \text { for } i=1, \ldots, m\right],
$$

where $C^{\prime}$ is the $(n+1) \times(n+1)$ symmetric matrix defined by

$$
C^{\prime}[i, j]=\left\{\begin{array}{l}
1 \quad \text { if } i=j=n+1 \\
0 \quad \text { otherwise }
\end{array}\right.
$$

and $A_{i}^{\prime}, i=1, \cdots, n$ is the $(n+1) \times(n+1)$ symmetric matrix defined by

$$
A_{i}^{\prime}=\left(\begin{array}{cc}
A_{i} & 0 \\
0 & b_{i}-\left\langle A_{i}, X_{0}\right\rangle
\end{array}\right)
$$

Finally, $X^{\prime}$ is the $(n+1) \times(n+1)$ matrix such that

$$
X^{\prime}=\left(\begin{array}{cc}
X & 0 \\
0 & \lambda
\end{array}\right)
$$


Choose some $X_{0} \in \operatorname{int}(\mathrm{K})$ (for instance the identity matrix). Then, $X^{\prime}=$ $\left(\begin{array}{cc}X_{0} & 0 \\ 0 & 1\end{array}\right)$ is a strictly feasible solution of $(A P)$. Apply the algorithm described in Section 3 to $(A P)$.

\section{Numerical tests}

The algorithm has been tested on some benchmark problems issued from the library of test problems SDPLIB [13]. We have taken $\rho=0.90$ and the stopping criterion $\epsilon=10^{-8}$. The first phase (phase 1) corresponds to the search of an initial strictly feasible solution and the second one (phase 2) is the resolution of the problem itself.

\begin{tabular}{|c|c|c|c|}
\hline Examples & $\begin{array}{c}\text { Size } \\
(\mathrm{m}, \mathrm{n})\end{array}$ & $\begin{array}{c}\text { Nbr. of iterations } \\
\text { Phase } 1\end{array}$ & $\begin{array}{c}\text { Nbr. of iterations } \\
\text { Phase 2 }\end{array}$ \\
\hline control1 & $(21,15)$ & 10 & 106 \\
\hline hinf1 & $(13,14)$ & 6 & 27 \\
\hline hinf2 & $(13,16)$ & 7 & 43 \\
\hline hinf3 & $(13,16)$ & 6 & 109 \\
\hline hinf4 & $(13,16)$ & 7 & 39 \\
\hline hinf5 & $(13,16)$ & 7 & 42 \\
\hline hinf7 & $(13,16)$ & 7 & 38 \\
\hline hinf9 & $(13,16)$ & 5 & 28 \\
\hline hinf10 & $(21,18)$ & 7 & 57 \\
\hline truss1 & $(6,13)$ & 15 & 17 \\
\hline truss4 & $(12,19)$ & 25 & 21 \\
\hline
\end{tabular}

We have also tested the infeasible problems infd1 and infd2 of SDPLIB. The algorithm concludes to their infeasibility.

Acknowledgements. The authors thank the referees for their careful reading and their precious comments and remarks. Their help has been much appreciated.

\section{REFERENCES}

[1] F. Alizadeh, Interior point methods in semidefinite programming with applications to combinatorial optimization. SIAM J. Optim. 5 (1995) 13-51.

[2] F. Alizadeh, J.P.A. Haeberly and M.L. Overton, Primal-dual interior point methods for semidefinite programming: convergence rates, stability and numerical results. SIAM J. Optim. 8 (1998) 746-768.

[3] S.J. Benson, Y. Ye and X. Zhang, Solving large-scale sparse semidefinite programs for combinatorial optimization. SIAM J. Optim. 10 (2000) 443-461. 
[4] P. Gahinet, A. Nemirovski, The projective method for solving linear matrix inequalities. Math. Program. 77 (1997) 163-190.

[5] C. Helmberg, F. Rendl, R.J. Vanderbei and H. Wolkowicz, An interior point method for semidefinite programming. SIAM J. Optim. 6 (1996) 342-361.

[6] M. Kojima, S. Shindoh and S. Hara, Interior point methods for the monotone semidefinite linear complementarity problem in symmetric matrices. SIAM J. Optim. 7 (1997) 86-125.

[7] Y. Nesterov and A. Nemirovski, Interior point polynomial algorithms in convex programming. SIAM Stud. Appl. Math. 13, Society for Industrial and applied Mathematics (SIAM), Philadelphia, PA (1994).

[8] A. Nemirovski and K. Scheinberg, Extension of Karmarkar's algorithm onto convex quadratically constrained quadratic problems. Math. Program. 72 (1996) 273-289.

[9] M. Overton and H. Wolkowicz, Semidefinite programming. Math. Program. 77 (1997) 105-109.

[10] M.V. Ramana, L. Tuncel and H. Wolkowicz, Strong duality for semidefinite programming. SIAM J. Optim. 7 (1997) 641-662.

[11] L. Vandenberghe and S. Boyd, Positive definite programming. SIAM Rev. 38 (1996) 49-95.

[12] H. Wolkowicz, G.-P.-H. Styan, Bounds for eigenvalues using traces. Linear Algebra Appl. 29 (1980) 471-506.

[13] http://infohost.nmt.edu/ sdplib/ 